synthesise actin destined for the cell surface, whereas actin for thin filaments does not accumulate.

These considerations require reevaluation of reports of the time during myogenesis when the messenger RNAs for myosin are transcribed and translated. If the presence of a molecule with the molecular weight of 200,000 is taken as evidence for the synthesis of myosin (Yaffe and Dym, Cold Spring Harb. Symp. quant. Biol., 37, 543: 1972) then all cells, including replicating presumptive myoblasts, must be transcribing and translating mRNAs for some kind of myosin. Accordingly it is not surprising that Rubinstein and colleagues (Biochem. biophys. Res. Commun., 57, 438; 1974) now report that myosin and actin are indeed coordinately synthesised and turned over, not only in myotubes but atso in mononucleated presumptive myoblasts, cartilage cells, fibroblasts and BUdR-suppressed myogenic cells.

Lastly, a smouldering controversy of considerable interest to molecular geneticists exists between those who claim that myoblasts withdraw from the cell cycle as a precondition to fusion, and those who claim that cessation of DNA synthesis results from fusion. The most emphatic statement of the second position is that of Doering and Fischman (Devl Biol., 36, 225; 1974). Based on what is termed a " $\%$ fusion" index they conclude that myogenic cells whose DNA synthesis is blocked by Ara-C fuse; according to these authors replicating myogenic cells have the option to replicate, or to fuse, and, as a consequence of fusing, cease replicating. Before these important conclusions can be accepted, however, it should be noted that: (1) Ara-C is routinely used to kill replicating cells, so that the design of the Doering and Fischman experiment must yield a spuriously high " $\%$, fusion" index; (2) early fusion cannot be measured with the light microscope; and (3) it is impossible to distinguish 'fibroblasts' from presumptive myoblasts. Given these uncertainties it will be interesting to see if those who speculate that myoblasts withdrew from the cell cycle as a precondition to fusion can devise more cogent experiments.

H. Holtzer

\section{Slaved disk model for Hercules X-1}

from James Pringle

A POSSIBLE solution to some of the morc puzzling aspects of the X-ray star Hercules X-1 has been proposed in a recent article by Roberts (Astrophys. J., $187,575 ; 1974)$. The $X$ rays from $\mathrm{Her}$ $\mathrm{X}-1$ are regularly pulsed with a period of $1.24 \mathrm{~s}$ and the source of $\mathrm{X}$ rays is one member of a binary star system which has an orbital period of $1.7 \mathrm{~d}$. The X-ray source is thought to be a neutron star with a strong dipole magnetic field and the $X$ rays are produced by material transferred from the 'normal' star of the binary system accreting on to the compact neutron star. Because of the strong magnetic field, the accreting material is channelled down on to the magnetic polar caps of the neutron star dissipating its kinetic energy of infall when it strikes the surface. So much energy is liberated in such a small region that the temperature rises to more than 1()$^{7} \mathrm{~K}$. Thus most of the radiation emerges as $X$ rays. The two magnetic polar caps are then two X-ray emitting hotspots: if the magnetic axis is offset from the rotation axis, rotation of the star with a $1.24 \mathrm{~s}$ period provides a natural explanation of why the observed $X$ rays are regularly pulsed.

Furthermore, the X-ray source displays a third periodicity. The $\mathrm{X}$ rays are visible for only about 10 to 12 consecutive days, reappearing regularly at 35-d intervals. Explanations of this phenomenon-for example, variable mass transfer or precession of the pulsing neutron star-have difficulty in accounting for another aspect of the problem. The 'normal' component of the binary system was identified in 1972 as the previously known variable star HZ Herculis. This star is so close to the luminous $\mathrm{X}$-ray source that X-ray heating causes one side of it to be about four times brighter than the other. The brightened face follows the neutron star around its orbit, and thus $\mathrm{HZ}$ Her can be observed to vary regularly in brightness with a period of $1.7 \mathrm{~d}$. The puzzling feature of this 1.7-d optical variation is that, although the $X$ rays can be seen for only 10 out of every $35 \mathrm{~d}$, the heating of the side of $\mathrm{HZ} \mathrm{Her}$ nearest to the X-ray source continues throughout the cycle, even when no $\mathrm{X}$ rays are observed.

Roberts' explanation of this is quite simple. He suggests that $\mathrm{HZ} \mathrm{Her}$ has its rotation axis at an angle to the orbital rotation axis. In this case, the rotation axis of HZ Her precesses about the orbital rotation axis with a period of about $35 \mathrm{~d}$. The material transferred by $\mathrm{HZ} \mathrm{Her} \mathrm{towards} \mathrm{its} \mathrm{companion} \mathrm{has}$ too much angular momentum to be able to fall on to it directly and, instead, forms an accretion disk around the neutron star. In the disk, viscosity transfers angular momentum outwards, allowing the material to spiral slowly inwards. Roberts notes that the disk, which is opaque to $X$ rays, will lie in the plane perpendicular to $\mathrm{HZ}$ Her's rotation axis, and thus in general not in the orbital plane of the system. Roberts names this the Slaved Orien- tated Disk, or SOD for short. Thus the angle between our line of sight to the $X$-ray source and the plane of the SOD varies with the $35-d$ precession period of $\mathrm{HZ} \mathrm{Her.} \mathrm{Since} \mathrm{the} \mathrm{X-ray} \mathrm{source} \mathrm{can}$ only be seen when this angle is sufficiently large, one expects the visibility of the $X$ rays to vary with the 35-d period. On the other hand, HZ Her is so close to the X-ray source that the (X-ray) shadow cast on its surface by the disk is quite small. Thus one expects the heating of $\mathrm{HZ}$ Her to continue, even when the disk temporarily obscures the X-ray source from observation.

This is by no means the whole story. Roberts also explains why the rotation axes might be expected to be misaligned and shows how his model can account for several further observational details. There are now a number of models which seem to account for the 35-d period of Her X-1 and its associated problems, but nobody has yet performed any detailed calculations. Roberts, however, excuses himself by quoting J. A. Wheeler's First Moral Principle: "Never do a calculation until you already know the answer".

\section{Asia as a composite continent}

\section{from Peter J. Smith}

Geomagnetism Correspondent

Whether or not it is realistic to suppose that any of the present continents could have formed by the accretion of separate crustal blocks, the important question of continental fusion continues to receive considerable attention. Most recently the debate has centred particularly on Africa (see, for example, Piper et al., Nature, 245, 244; 1973) with new evidence suggesting that the orogenic belts between the major cratonic areas of that continent may not be sutural but more probably formed in situ, although the idea of what Kropotkin (Eos, 53, 180; 1972) has termed the "composite continent" was actually first applied to Asia by Argand (Coll. Rep. 13th Int. Geol. Congr. $1922,1,171 ; 1924)$ just 50 years ago and long before the new global tectonics had been thought of.

Since then, evidence supporting the composite nature of Asia has been adduced from palaeomagnetism, palaeontology, geology and petrotectonics. In a useful and commendably concise article Burrett (Earth planet. Sci. Lett., 21, 181; 1974) has now brought all these data together to delineate the Asian block boundaries and to estimate the timings of the various collisions thought to be involved. Although the field information is not entirely clear in all cases, there seem to be about nine blocks present; and Burrett sum- 